\title{
Bundesrätlicher Tarifeingriff hat weitreichende Konsequenzen
}

\author{
Christian Oeschger \\ Wissenschaftlicher Mitarbeiter, Abteilung Ambulante Versorgung und Tarife
}

\begin{abstract}
Zum zweiten Mal greift der Bundesrat in die TARMED-Tarifstruktur ein. Die Vernehmlassung der Verordnung ist bereits im Gange. Erste Analysen der FMH zeigen ein düsteres Bild: Vor allem im operativen und invasiven Bereich sind die Kürzungen teils massiv - aber auch andere Disziplinen (Spezialisten) leiden stark. Die FMH lehnt isolierte Eingriffe in die bestehende Tarifstruktur ab und verfolgt mit dem Projekt TARCO weiterhin das Ziel einer Gesamtrevision.
\end{abstract}

Der Bundesrat greift per 1. Januar 2018 zum zweiten Mal nach 2014 in die TARMED-Tarifstruktur ein. Am 22. März 2017 hat er anlässlich einer Medienkonferenz in Bern die Vernehmlassung dazu gestartet. Der Bundesrat plant einen wesentlich umfangreicheren Eingriff als 2014. Der zweite Eingriff wird die freie Praxis und die Spitalambulatorien finanziell teils erheblich treffen. Nebst der Einführung zusätzlicher Abrechnungsregeln plant der Bundesrat direkte Eingriffe in die Tarifierung. Die FMH hat sämtliche Unterlagen im Rahmen der Vernehmlassung auf ihrer Website aufgeschaltet: www. fmh.ch $\rightarrow$ Ambulante Tarife $\rightarrow$ TARMED Tarif $\rightarrow$ TARMED Änderungen ab 1.1.2018.

\section{Die verordneten Massnahmen im Detail}

Die Massnahmen sind sehr umfangreich und komplex, welche das Bundesamt für Gesundheit (BAG) dem Bundesrat vorgeschlagen hat. Sie basieren auf dem aktuell gültigen Tarif, der bereits im Jahr 2014 in einigen Kapiteln um 8,5 Prozent gesenkt wurde. Es kommt damit zu weiteren Absenkungen.

\section{Tarifdelegierten-Tag}

Am 20. April 2017 fand in Bern derTarifdelegierten-Tag der FMH statt, zu welchem alle Präsidentinnen und Präsidenten sowie Tarifdelegierten der in der Ärztekammer vertretenen Organisationen eingeladen waren. Die Veranstaltung ist mit über 80 Teilnehmenden wiederum auf grosses Interesse gestossen. Die Präsentationen von Dr. med. Urs Stoffel, Dr. med. Susanne Christen, Kerstin Schutz, Sabine Zehnder, Thomas Kessler und Christian Oeschger finden Tarifdelegierte ab sofort im Ablagebereich von myFMH:Tarifdelegierten-Tag - Journée des délégués tarifaires $\rightarrow$ 20.04.2017
Das BAG schlägt verschiedene Kategorien von Anpassungen vor. Die für die Ärzteschaft wichtigen Punkte sind nachfolgend aufgeführt:

Die Massnahmen der ersten Kategorie sehen unter anderem vor, die heute geltenden «Quantitativen Dignitäten» über den gesamten Tarif einzuebnen. Die heute zwischen 0,905 (FMH5) und 2,2625 (FMH12) liegenden Werte sollen auf 0,968 nivelliert werden. Die «Quantitativen Dignitäten» sind neben dem Referenzeinkommen, der Jahresarbeitszeit und der «Ärztlichen Produktivität» entscheidend für den Taxpunkt der «Ärztlichen Leistung (AL)». Für Leistungen mit einer "Quantitativen Dignität» von FMH-5 bedeutet diese Massnahme eine leichte Aufwertung, bei allen anderen Leistungen ist mit massiven Kürzungen zu rechnen. Der praktische Arzt mit einer dreijährigen Ausbildung wird sogar auf 0,940 abgesenkt.

Des Weiteren will der Bundesrat auch an der «Ärztlichen Produktivität» schrauben. Sie soll für alle OP-Leistungen angehoben werden. Diese Massnahme wirkt sich ebenfalls - teils erheblich - auf die AL aus und senkt sie weiter ab.

Der Bundesrat greift auch auf der «Technischen Leistung (TL)» ein. So werden die Kostensätze für viele Sparten um 10 Prozent abgesenkt und somit auch der TL-Taxpunkt.

Zu einschneidenden Kürzungen kommt es ausserdem in spezifischen Bereichen des Tarifs: Im Kapitel der Bildgebung schlägt das BAG vor, die AL für MRI- und CTLeistungen gänzlich zu streichen und den Arzt nur noch für die Befundung der CT- und MRI-Bilder zu entschädigen. Bei Tarifpositionen aus dem Bereich der Kataraktoperation, der intravitrealen Injektion, des Belastungsund Holter-EKG, der Koloskopie und der stereotaktischen 
Radiotherapie beinhaltet der BAG-Vorschlag, Leistungen entweder gänzlich zu streichen oder aber die Minutagen stark abzusenken.

In der zweiten Kategorie von Massnahmen ist vor allem die Einführung von Abrechnungslimitationen einschneidend. Das BAG schlägt vor, gewisse Untersuchungen durch den Facharzt von Handlungsleistungen in Zeitleistungen umzuwandeln und diese in der Menge, die abgerechnet werden kann, sowohl auf Ebene der einzelnen Sitzung als auch für ein Zeitintervall zu limitieren. Ähnlich verhält es sich bei Leistungen in Abwesenheit des Patienten: Diese werden zwar weiter differenziert, aber ebenfalls limitiert.

Die dritte Kategorie von Massnahmen beinhaltet die Aufhebung des Zuschlags von 10\% auf Verbrauchsmaterialien und Implantate (Generelle Interpretation 20) sowie die Elimination von Tarifpositionen für stationäre Leistungen und Leistungen der Unfall-, Invaliden- oder Militärversicherung sowie alle Verweise auf das entsprechende Gesetz.

Detaillierte Informationen zu den einzelnen Massnahmen finden FMH-Mitglieder auf myFMH: https:// myfmh.fmh.ch/ $\rightarrow$ Vernehmlassung Tarifeingriff Consultation intervention tarifaire

\section{Massive Auswirkungen auf die "Ärztliche Leistung"}

Eine umfassende und differenzierte Beurteilung der Massnahmen und deren Auswirkungen ist für die FMH erst nach weiteren internen Analysen sowie Simulationen machbar. Diese werden zu gegebener Zeit kommuniziert.

Schon heute aber ist klar, dass das umfangreiche Massnahmenpaket des zweiten Tarifeingriffes die Mitglieder der FMH teils erheblich finanziell treffen wird. Alleine die Auswirkungen des Eingriffs aus den «Quantitativen Dignitäten" sind bei einigen Disziplinen massiv. Hinzu kommen die Erhöhung der Ärztlichen Produktivität bei sämtlichen operativen Eingriffen, die konkrete Korrektur von Minutagen bei ausgewählten Tarifpositionen sowie die neu gültigen Limitationen auf einigen wichtigen Zeitleistungen.

Zahlen der FMH und der Ärztekasse zeigen, dass es vor allem im Bereich der nichtärztlichen Löhne seit Einführung des TARMED zu wesentlichen Steigerungen gekommen ist, welche bisher nie angepasst worden sind. Mit dem technischen Fortschritt und der demographischen Entwicklung ist der Personalbedarf in der Praxis stark angestiegen. Zudem konnten seit 2014 auch die Spitalärzte einen Lohnzuwachs verzeichnen. Wenn es attraktiv bleiben sein soll, nach der Weiterbil- dungszeit am Spital in die freie Praxis zu wechseln, muss es möglich sein, als freipraktizierender Arzt ein konkurrenzfähiges Einkommen generieren zu können. Mit einseitigen Einsparungen, wie sie das BAG nun vorschlägt, ist dies nicht mehr möglich.

\section{Umfassende Gesamtrevision statt punktuelle Eingriffe}

Unbestritten ist von der FMH und der in der Ärztekammer vertretenen Organisationen, dass die im Jahr 2004 eingeführte national einheitliche ambulante Tarifstruktur veraltet und deshalb zu revidieren ist. Die FMH lehnt punktuelle Eingriffe in die bestehende Tarifstruktur jedoch ab, da sie zu weiteren Verzerrungen in der heutigen gültigen Tarifstruktur führen. Nach der Ablehnung des ersten Revisionsvorschlages in der Urabstimmung im Mai 2016 hat die FMH deshalb das Revisionsprojekt TARCO zur Nachbesserung gestartet. TARCO bezweckt eine sachgerechte und betriebswirtschaftliche Tarifierung aller ambulanten ärztlichen Leistungen. Nur eine umfassende Tarifrevision gemeinsam mit allen Ärzteorganisationen und Tarifpartnern ist aus Sicht der Ärzteschaft der einzig richtige Weg.

\section{Nehmen Sie Ihre Rechte wahr}

Auch wer nicht zum Vernehmlassungsverfahren eingeladen wird, kann sich zu einer Vorlage äussern. Die Vernehmlassung läuft bis 21. Juni 2017. Nehmen Sie als Fachgesellschaft Ihr Recht wahr und geben Sie eine Stellungnahme ab zur Verordnung. Die Stellungnahme ist elektronisch oder per Post an folgende Adresse zu senden

Bundesamt für Gesundheit BAG

Direktionsbereich Kranken- und Unfallversicherung

Abteilung Leistungen

3003 Bern

abteilung-leistungen[at]bag.admin.ch

Tel. +4158462 2111

Bitte senden Sie eine Kopie Ihrer Stellungnahme an die FMH, damit wir Ihre Argumente in die konsolidierte Vernehmlassungsantwort einbeziehen können. Um genügend Vorlaufzeit zu haben, sind wir Ihnen dankbar für die Einreichung bis spätestens 1. Juni 2017 an folgende Adresse:

$\mathrm{FMH}$

Abteilung Ambulante Versorgung und Tarife

Frohburgstrasse 15

4600 Olten

tarife.ambulant[at]fmh.ch

Tel. +41313501230 


\begin{tabular}{ll}
\hline Zeitplan & \\
\hline 22.03 .2017 & Start der Vernehmlassung \\
\hline 29.03 .2017 & Veröffentlichung der Erstbeurteilung der FMH \\
\hline $29.03 .2017-$ & Internes Zeitfenster für umfassende \\
17.04 .2017 & Analysen der FMH \\
\hline $18.04 .2017-$ & $\begin{array}{l}\text { Zeitfenster für umfassende Analysen der FMH } \\
\text { für ihre angeschlossenen Fachgesellschaften } \\
\text { und kantonale Ärztegesellschaften }\end{array}$ \\
\hline $15.05 .2017-$ & $\begin{array}{l}\text { Zeitfenster für Volumis online: eigenständige } \\
\text { Analyse für FMH-Mitglieder }\end{array}$ \\
\hline 01.06 .2017 & $\begin{array}{l}\text { Einsendeschluss für Einreichung der Stellung- } \\
\text { nahmen der Fachgesellschaften bei der FMH }\end{array}$ \\
\hline 21.06 .2017 & $\begin{array}{l}\text { Ende der Vernehmlassung } \\
\text { Einreichung der FMH-Stellungnahme } \\
\text { beim BAG }\end{array}$ \\
\hline 01.01 .2018 & $\begin{array}{l}\text { Inkrafttreten der Verordnung (zweiter Tarif- } \\
\text { eingriff) }\end{array}$ \\
\hline
\end{tabular}

\section{Wir unterstützen Sie und Ihre Fachgesellschaft}

Das Departement Ambulante Versorgung und Tarife der FMH und NewIndex unterstützen Sie gerne bei Analysen für Ihre eigene Stellungnahme zuhanden des Bundesamtes für Gesundheit. Kontaktieren Sie uns unter tarife.ambulant[at]fmh.ch oder info[at]newindex.ch. Wir liefern Ihnen eine ausführliche Analyse der Vorlage, basierend auf dem Mengengerüst Ihrer Fachgesell-

Korrespondenz:

FMH / Abteilung Ambulante Versorgung und Tarife Frohburgstrasse 15

CH-4600 Olten

Tel. 0313591230

Fax 0313591238

tarife.ambulant[at]fmh.ch schaft oder Ihrer kantonalen Gesellschaft. Wir beraten aber auch Sie als Einzelmitglied: In den kommenden Wochen finden Sie dazu Informationen auf unserer Website. 